\title{
Online Dynamic Travel Time Prediction using Speed and Flow Measurements
}

\author{
Luis Leon Ojeda*
}

\author{
Alain Y. Kibangou**
}

\author{
Carlos Canudas de Wit***
}

\begin{abstract}
Traffic forecasting is considered nowadays as one of the most important traffic management techniques on road networks. To provide suitable control strategies and advanced traveler information, which improve traffic performance, a continuous short-term prediction is a significant requirement. In this paper, we propose a new approach for travel time forecasting between two points of interest of a given highway divided in nodes and links. Since nodes and links have distinct characteristics, two different prediction methods are proposed. The resulting predicted travel time is then computed as the sum of predicted travel times in nodes with those in links. An adaptive Kalman filtering approach is considered for predicting sojourn time in nodes and flows at boundaries of links. Inside links, divided in cells for improving resolution, a deterministic observer is used for computing unmeasured densities. The performance of the proposed method is evaluated by using data of the Grenoble south ring, a case study of the NoE Hycon2.
\end{abstract}

\section{INTRODUCTION}

Travel time can be defined as the time needed to traverse a road between two points of interest. It is one of the most used traffic index. It is particularly useful for a wide variety of users including transportation engineers, planners, and consumers, [1], [2].

Multi-step ahead dynamic travel time forecasting has been widely investigated in the literature, see for instance the survey [3]. It has been pointed out that travel time forecasting is firmly related to the appropriate available data, and the arise of forecasting methodologies is due to this available information. This index can be predicted either by means of direct measurements of travel time, or through inference from indirect measurements such as speed or flows.

Direct travel time measurements can be obtained using license plate matching techniques, probe vehicles, and, in general, floating car (FCD) or mobile data (FMD) [4]-[7]. Nevertheless, those methodologies present some limitations, such as, special requirements needed for installation, driver's privacy issues, and limited available data, among others.

Indirect measurements are greatly more available. They can be exploited following two different strategies. The first corresponds to the case where both flow, occupancy, and speed measurements are available, whereas in the second only flow and occupancy measurements are attainable.

* INRIA Rhône-Alpes, NeCS team, Grenoble, France (e-mail: luis.leon@inria.fr)

** Gipsa-Lab, University Joseph Fourier, CNRS, NeCS team, Grenoble, France (e-mail: alain.kibangou@ujf-grenoble.fr)

*** Gipsa-Lab, CNRS, NeCS team, Grenoble, France (e-mail carlos.canudas-de-wit@gipsa-lab.grenoble-inp.fr)
For the first case, the problem reduces to the prediction of the travel time from the velocity measurements. Several works have been presented following this direction, see [8][10]. The main advantage of this approach comes from its simple online implementable nature. However it presents the disadvantage that between two consecutive detectors a mean speed is assumed, therefore the more the sensors are spaced, the less the results are accurate.

As for the second case, two approaches can be used to estimate the travel time; computing a mean speed from the flow and occupancy measurements, [10]-[12], or by estimating travel time from density, making use of only flow measurements. For the latter the density is estimated from the vehicle conservation law [13], and the common methodology to obtain the future values of the travel time is through the prediction of the boundary flows. Note that for achieving the flow prediction several approaches, such as, time series models, Kalman filtering, among others, can be used. In [10] for instance, the dynamic travel time estimation was achieved under the prediction of traffic flow and occupancy using seasonal ARIMA model together with a Kalman filter approach. Also here however, distance between two consecutive sensors is not really taken into account, generating a low accuracy in the forecasting results when the distance between sensors is important enough.

Compared with the previous works, the one presented herein aims to develop a robust, accurate, and easy online implementable prediction methodology.

For this, two highway sections need to be treated differently: links, consisting on a concatenation of cells, and nodes, portion with an input or an output. For links the travel time forecasting will be achieved by making use of a Luenberger state observer based on the conservation law principle, whose inputs are boundary traffic flows predicted using an adaptive Kalman filter (AKF) approach. This is, to the best of our knowledge, the first time where AKF and CTM-based predictor are used together to achieve this goal. When considering nodes, on the other hand, we will assume that speed measurements are available. Thus, after computing the time spent in the node up to the current time, we can apply an adaptive Kalman filter approach to predict its future values.

This paper is organized as follows, first in section II we introduce our problem formulation, in section III we present 
an adaptive Kalman filter approach for multi-step ahead time series prediction, applied for boundary flows and node sojourn time. In section IV the study of node sojourn time and travel time prediction in links is conducted. Simulation results will be presented in section $\mathrm{V}$, and then in section VI we present our conclusions.

\section{PROBLEM FORMULATION}

Given a section of interest in the highway, from point $\mathrm{A}$ to $\mathrm{B}$, historical data of the boundary traffic flows $\varphi_{t, d}^{u}, \varphi_{t, d}^{d}$ and mean speed inside the nodes $v_{t, d}^{\text {mean }}, t=0,1, \cdots, T$, $d=1,2, \cdots, D-1$, and current information until time $t_{0}$ of the day $D,\left\{\varphi_{t, d}^{u}\right\},\left\{\varphi_{t, d}^{d}\right\},\left\{v_{t, d}^{\text {mean }}\right\}, t=0,1, \cdots, t_{0}$. Our aim is to predict the travel time at time $t=t_{0}+1, \cdots, t_{0}+$ $\Delta T$ from A to B (see figure 1).

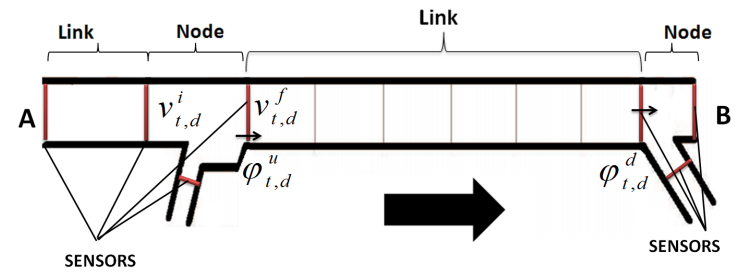

Fig. 1. Architecture of a section of a highway

As depicted in figure 1, a section is composed of links and nodes. A link is defined as a concatenation of homogeneous cells, i.e. cells with the same number of lanes. While a node is a portion of the ring-road with an on-ramp of off-ramp. The predicted section travel time $T_{t}^{A \rightarrow B}$ is then computed as:

$$
T_{t}^{A \rightarrow B}=\sum_{i=1}^{k} T_{t_{i-1}}^{i}
$$

with $k$ the number of elements present in the section, cells plus nodes, $T^{i}$ the predicted travel time for the $i_{t h}$ element, and $t_{i-1}$ the time a vehicle enters this element.

Our problem then reduces to predict $T_{t}$ in a node or in a link given current and historical measurements of flows and velocities. The overall forecasting scheme is depicted in figure 2 .

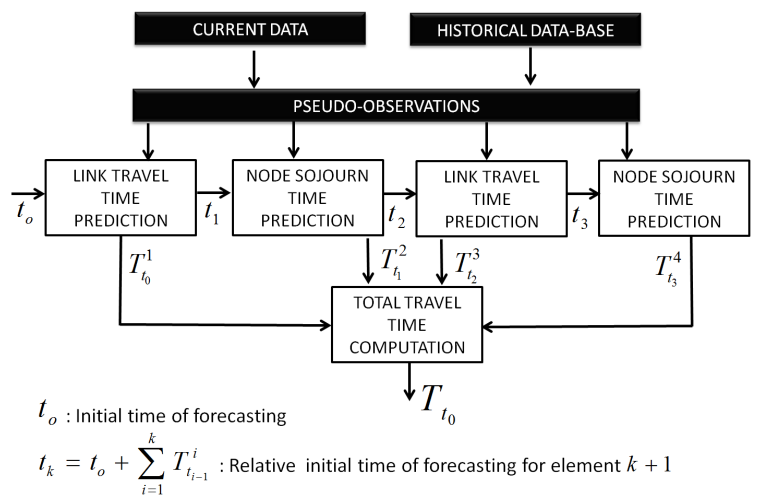

Fig. 2. Short-term total travel time prediction scheme.
As it was aforementioned, the travel time is computed according to the available data. For a cell it can be defined as:

$$
T_{t}=f_{t} \Delta x
$$

where $\Delta x$ stands for the cell length and $f_{t}$ has the dimension of the unit time for crossing the cell. If speed measurements are available then $f_{t}$ is simply the inverse of the cell mean speed $v_{t}^{\text {mean }}$. Otherwise,

$$
f_{t}=\frac{\rho_{t}}{\varphi_{t}^{u}+\varphi_{t}^{d}}
$$

where $\varphi_{t}^{u}, \varphi_{t}^{d}$, and $\rho_{t}$ stand for upstream and downstream cell boundary flow, and cell density respectively.

Therefore for nodes the travel time is directly given by (2) with $f_{t}=\frac{1}{v_{t}^{\text {mean }}}$, where the node mean velocity is defined as $v_{t}^{\text {mean }}=\frac{v_{t}^{i}+v_{t}^{f}}{2}($ see figure 1$)$.

While for a link of $\mathrm{n}$ cells, the travel time at $t_{0}$ can be computed as:

$$
T_{t o}=\sum_{i=1}^{n} T_{t_{i-1}}^{i}=\sum_{i=1}^{n} \Delta x\left(\frac{\rho_{t_{i-1}}^{i}}{\varphi_{t_{i-1}}^{u_{i}}+\varphi_{t_{i-1}}^{d_{i}}}\right)
$$

with $T^{i}$ the travel time of the $i_{t h}$ cell and $t_{i}=t_{i-1}+T_{t_{i-1}}^{i}$ the relative current time of the $i_{t h}+1$ cell. It is worth noting that by dividing the link in several small cells, and thus predicting the travel time for each one, rather than directly predicting this for the complete link, a higher accuracy can be obtained, since inside a link the traffic dynamics can vary substantially in the presence of a congestion wave.

\section{AdAPtive Kalman FILTERING STRATEGY}

This methodology focuses on the development of a multistep ahead forecasting approach that, under an online implementable algorithm, can achieve accurate prediction performance with no requirement of extensive data calibration nor large historical database.

We are aiming to obtain a multi-step ahead predictor based on Kalman approach that could also be able to capture eventual atypical events between the current and historical data. For this reason we transform our prediction problem to a filtering one, where the measurements are pseudoobservations obtained from the predictor proposed in [14].

One of the problems that emerges from this strategy is the hypothesis of a priori knowledge of noise covariance matrices. for this, we estimate online and empirically theses matrices using the approach proposed in [15].

This AKF approach was first proposed in [16], and it was oriented towards demand flow forecasting. However, it can be extended to any time series when heuristics are available. In the sequel we describe an extension of [16].

Let us consider the following Gauss-Markov model:

$$
\begin{aligned}
& x_{t}=x_{t-1}+w_{t} \\
& y_{t}=H_{t} x_{t}+v_{t}
\end{aligned}
$$

where $w_{t} \sim \mathcal{N}\left(q_{t}, Q_{t}\right)$ and $v_{t} \sim \mathcal{N}(0, R)$ are mutually independent and independent from the initial conditions of the state $x_{t}$. We denote by $y_{0: t}$ the set of measurements up to time $t$, as aforementioned this set will be composed by 
pseudo-observations obtained from the approach described [14].

The methodology in [14] combines a constant and a nonparametric predictor. The constant one acts for small prediction horizon, while, with the increasing of the horizon, heuristics are used for prediction. Based on this consideration, the following strategy was proposed:

$$
\hat{y}_{t_{0}+t}=y_{t_{0}+t}^{H}+K \Delta y_{t_{0}}
$$

with

$$
\begin{aligned}
& \Delta y_{t_{0}}=y_{t_{0}}-y_{t_{0}}^{H} \\
& K=\left\{\begin{array}{ccc}
\eta\left(1-\frac{t}{\Delta t_{\max }}\right), & \text { if } & 0<t \leq \Delta t_{\max } \\
0, & \text { if } & t>\Delta t_{\max }
\end{array}\right.
\end{aligned}
$$

$y_{t}^{H}$ being the level of the averaged historical data, $\eta$ being the weighting of current and historical data, and $\Delta t_{\max }$ the maximum horizon for the constant predictor.

The mean and the variance of both process and observation noises are estimated with the available data using the approach proposed in [15]. Considering a time window of $N$ past samples, the method acts as follows:

- Initialization:

$\hat{x}_{0 \mid 0}=x_{t_{0}}, P_{0 \mid 0}$ is initialized with a suitable positive value,

$$
\begin{aligned}
& \hat{r}=\frac{1}{N} \sum_{t=t_{0}-N+1}^{t_{0}}\left(y_{t}-H_{t} x_{t}\right), \\
& R=\frac{1}{N-1} \sum_{t=t_{0}-N+1}^{t_{0}}\left(y_{t}-H_{t} x_{t}-\hat{r}\right)^{2}, \\
& \hat{q}_{0}=\frac{1}{N} \sum_{t=t_{0}-N+1}^{t_{0}}\left(x_{t}-x_{t-1}\right), \\
& \hat{Q}_{o}=\frac{1}{N-1} \sum_{t=t_{0}-N+1}^{t_{0}}\left(x_{t}-x_{t-1}-\hat{q}_{0}\right)^{2} .
\end{aligned}
$$

- Prediction process: for $t=t_{0}+1, \cdots, t_{0}+\Delta T$

- Kalman filtering equations:

$$
\hat{x}_{t \mid t}=\left(I-K_{t} H_{t}\right) \hat{x}_{t-1 \mid t-1}+K_{t} y_{t}+\left(I-K_{t} H_{t}\right) q_{t}
$$

with

$$
\begin{gathered}
K_{t}=P_{t \mid t-1} H_{t}^{T}\left(H_{t} P_{t \mid t-1} H_{t}^{T}+R\right)^{-1} \\
P_{t \mid t}=\left(I-K_{t} H_{t}\right) P_{t \mid t-1}
\end{gathered}
$$

and

$$
P_{t \mid t-1}=P_{t-1 \mid t-1}+Q_{t-1} .
$$

- If $t>t_{0}+N$, the mean and the variance of the process noise are updated as

$$
\begin{gathered}
\hat{q}_{t}=\frac{1}{N} \sum_{t=1}^{N} q_{t} \\
\hat{Q}_{t}=\frac{1}{N-1} \sum_{t=1}^{N}\left(\left(q_{t}-\hat{q}\right)\left(q_{t}-\hat{q}\right)^{T}-\right. \\
\left.-\frac{(N-1)}{N}\left(P_{t-1 \mid t-1}-P_{t \mid t}\right)\right)
\end{gathered}
$$

$q_{t}=\hat{x}_{t \mid t}-\hat{x}_{t-1 \mid t-1}$ is an approximation of the process noise. See [15] for more details.

\section{TRAVEL TIME PREDICTION}

In this section, we now describe the travel time prediction by first considering links and then nodes.

\section{A. Link travel time prediction}

The objective here is the prediction of flows at the link boundaries and densities in each cell. For this, we refer to figure 3 , where a short-term traffic flow prediction approach is needed in order to obtain the predicted cell's densities, and consequently the predicted travel time.

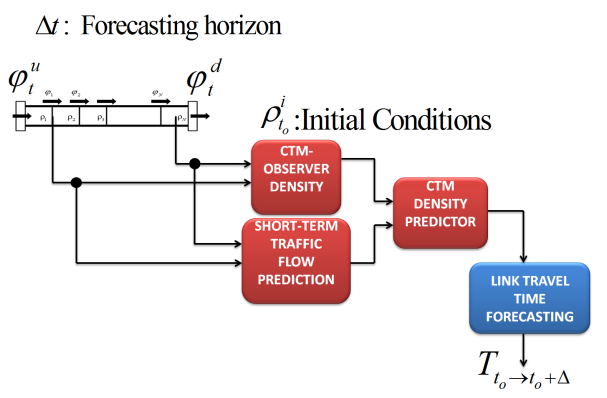

Fig. 3. Scheme of link travel time prediction.

In the sequel, we first present the strategy to accomplish the boundary flow prediction by introducing the state and measurement model in order to apply the approach introduced in section III. Second, we make use of the CTMbased state observer developed in [13], [17], for carrying out densities and interface flows prediction.

1) Boundary flows prediction: As aforementioned, we transform the prediction problem to an estimation one by using pseudo-observations described in section III. Given the vehicle conservation law constraint, the prediction of the link's boundary flows must not be unrelated. By introducing another state variable $\varepsilon_{t}=\varphi_{t}^{u}-\varphi_{t}^{d}$, the difference in time of the upstream and downstream flows, and therefore predicting $\varepsilon_{t}$ and $\varphi_{t}^{u}$ using historical data, we could impose some relation between the flows.

The model proposed is as follows:

$$
\begin{aligned}
x_{t} & =x_{t-1}+w_{t} \\
y_{t} & =H x_{t}+v_{t}
\end{aligned}
$$

$\begin{aligned} \text { where } x_{t} & =\left(\begin{array}{c}\varphi_{t}^{u} \\ \varepsilon_{t}\end{array}\right), w_{t}=\left(\begin{array}{c}w_{t}^{\varphi} \\ w_{t}^{\varepsilon}\end{array}\right), y_{t}= \\ \left(\begin{array}{c}y_{t}^{\varphi_{u}} \\ y_{t}^{\varphi_{d}}\end{array}\right), v_{t} & =\left(\begin{array}{c}v_{t}^{\varphi}-\hat{r}^{\varphi} \\ v_{t}^{\varepsilon}-\hat{r}^{\varepsilon}\end{array}\right), H=\left(\begin{array}{cc}1 & 0 \\ 1 & -1\end{array}\right)\end{aligned}$

The process and observation noises are modelled as $w_{t}^{\varphi} \sim$ $\mathcal{N}\left(0, Q_{t}^{\varphi}\right), w_{t}^{\varepsilon} \sim \mathcal{N}\left(0, Q_{t}^{\varepsilon}\right), v^{\varphi}{ }_{t} \sim \mathcal{N}\left(r^{\varphi}, R^{\varphi}\right)$, and $v_{t}^{\varepsilon} \sim$ $\mathcal{N}\left(r^{\varepsilon}, R^{\varepsilon}\right)$. As before, $y_{t}$ is the output of the Chrobok predictor, and $\hat{r}^{\varphi}$ and $\hat{r}^{\varepsilon}$ being the estimated observation noise sample mean for the upstream flow and the difference in time of upstream and downstream flows respectively, computed empirically using the strategy in [15].

For the prediction process the Kalman filtering equations (8)-(13) are to be applied to state-space model (14). 
2) Densities and interface flows prediction: It was aforementioned that the links will be divided into several cells in order to improve the accuracy of the density prediction. However it is clear that only boundary flows measurements are available, since sensors are located at node level, i.e. at the boundary of the links. Therefore, given the predicted boundary flows, our problem becomes an estimation problem, where the main objective is to reconstruct the densities inside the cells (not equipped with sensors). For this purpose we will make use of the observer developed by the authors in [13].

The macroscopic traffic flow model used to model the traffic dynamics is the cell transmission model (CTM):

$$
\rho_{t+1}^{i}=\rho_{t}^{i}+\frac{T}{l_{i}}\left(\varphi_{t}^{i}-\varphi_{t}^{i+1}\right)
$$

where $\rho_{t}^{i}$, is the predicted density of the $i_{t h}$ cell in a link at a specific time indexed by $t$, and $\varphi_{t}^{i}$ is the predicted flow at the cell interface. To guarantee convergence of (15) the condition $v_{i} T<l_{i}$ is sufficient [18] , where $T$ is the discrete time interval, $l_{i}$ the cell length, and $v_{i}$ the free-flow speed .

The interface flows in the CTM-model are computed as the minimum of the supply $S^{i}$, and the demand $D^{i-1}$ at each interface

$$
\varphi^{i}=\min \left\{D^{i-1}, S^{i}\right\}
$$

with

$$
\begin{aligned}
D^{i-1} & =\min \left\{v_{i-1} \rho^{i-1}, \varphi_{m, i-1}\right\}, \\
S^{i} & =\min \left\{\varphi_{m, i}, w_{i}\left(\rho_{m, i}-\rho^{i}\right)\right\}
\end{aligned}
$$

where $\varphi_{m, i}$ is the maximum flow allowed by the capacity of cell $i, \rho_{m, i}$ is the jam density (i.e. the maximum density that can be reached), $v_{i}$ corresponds to the free flow speed, and $w_{i}$ is the congestion wave speed in cell $i$.

The model (15) has different operation modes, according to the minimum between its upstream demand $D^{i-1}$ and its downstream supply $S^{i}$, the number of possible modes for a N cell link are: $M=2^{(N+1)}$ (figure 4). To constrain the model two assumptions are made, only one congestion wave may exist in a highway link, and that it will propagate upstream. With this, the number of modes is reduced to $M=2(N+1)$.

A deeper study of how the observer was built, as well as an exact definition of the system's dynamic matrices can be found in [13], [17].

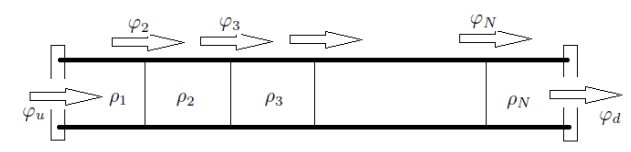

Fig. 4. Densities and flows in a link.

\section{B. Node sojourn time}

As shown in figures 2 and 5, our aim is to predict future values of the node sojourn time given velocity measurements (current and historical).

For this, we transform the prediction problem to an estimation one, as explained in section III, by considering the following model:

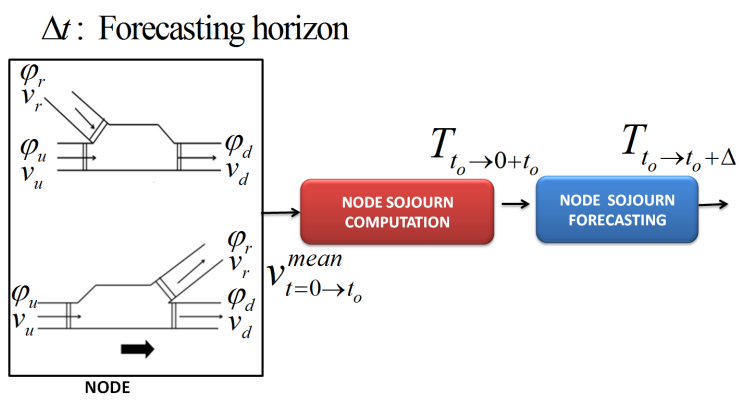

Fig. 5. Scheme of node sojourn time prediction.

$$
\begin{gathered}
x_{t}=x_{t-1}+w_{t} \\
y_{t}=x_{t}+v_{t}
\end{gathered}
$$

where $x_{t}=T_{t}, v_{t}=v^{T}-\hat{r}$.

The process and observation noises are modelled as $w_{t} \sim$ $\mathcal{N}\left(0, Q_{t}\right)$ and $v_{t} \sim \mathcal{N}(r, R)$. Where $y_{t}$ is the output of the Chrobok predictor, and $\hat{r}$ being the estimated noise sample mean computed empirically using the strategy proposed in [15].

It is worth noting that even though the system observations correspond with velocity measurements, we pre-process these data in order to get the node sojourn time, using the mean velocity above described.

For the prediction process the Kalman filtering equations (8)-(13) are to be applied to state-space model (17).

\section{Short-term total travel time prediction scheme}

The total travel time prediction, given by (1), is summarized in figures (2),(3), and (5). It is worth noticing that this methodology not only allows to predict the travel time between two points of interest, but also velocity conditions along the section with a high degree of granularity.

\section{Study CASE}

\section{A. Description of the Grenoble south ring}

The Grenoble south ring showcase has been already introduced elsewhere, see [13]. This is an approximately $10.5 \mathrm{~km}$ long highway constituted by two carriageways of two lanes each. Only the direction east-west was modelled. In this section there are 10 on-ramps and 7 off-ramps. This study case was implemented on the traffic simulator Aimsun, using real demand flow data introduced in the 13 entrances of the highway. The cells length and consequently the simulation time-step as well as the CTM parameters identification strategy were the same as adopted in [13].

The points of interested $\mathrm{A}$ and $\mathrm{B}$ on the highway can be depicted in figure 6, corresponding with the entrance Meylan and the exit Le rondeau. The scenario under consideration corresponds with the example that a vehicle wants to enter the highway through Meylan and traverse the entire highway. For this section there are 17 nodes and 9 links.

The traffic flows and velocities used to evaluate the goodness of the strategy proposed in this paper were obtained 


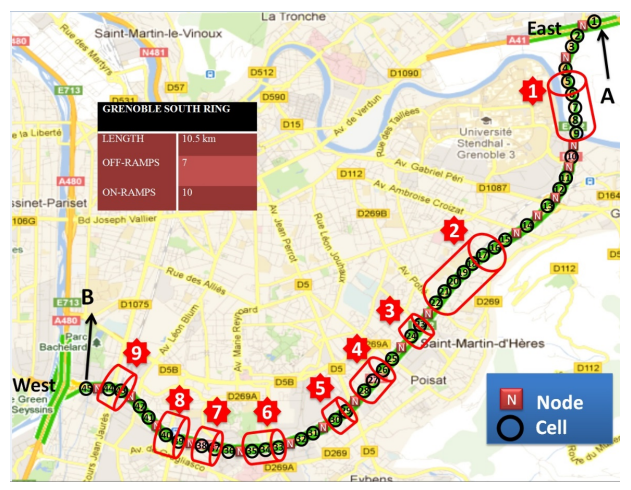

Fig. 6. Grenoble sounth ring show case.

using actual measurements of the Grenoble south ring resampled in a window of 24 hours. Due to the lack of historical database for either flow data and velocity in the nodes, a database of heuristics was created, by adding white noise to the current profile and filtering the resulting data with lowpass filters whose parameters were randomly generated.

\section{B. Simulation results}

As results we will present two different scenarios. The first corresponds at 7:45 am and the second at 19:00, both for a prediction horizon of 45 minutes, at steps of 5 minutes. Considering the same set of data as used in [13], the two cases were chosen in the morning and afternoon time, because of their level of congestion.

Both of the scenario not only will be provided with the total predicted travel time, but also with the trajectory of a vehicle when it moves through the predicted velocity conditions at different departing times.

In order to comply with the stability condition [18] the simulation step (sample time) was chosen to be 8s (see [13]). For the links and the nodes, the boundary flows and travel time were predicted every minute, thus they needed to be resampled back to $8 \mathrm{~s}$. Note also that the data were filtered using a 1st-order Butterworth low-pass filter with a cut-off frequency of $10 \mathrm{mHz}$.

Figure 7 shows the forecasting result at a current time of 07:45 am. Although it is seen that better results are obtained when the prognosis horizon is smaller, a fairly good match between the predicted and measured travel time is observed. The length of the section is $10.5 \mathrm{~km}$ long. Therefore if a passenger desires to leave at 07:45 am the results show that 25 minutes are needed to traverse this section, that corresponds with a average velocity of $25 \mathrm{~km} / \mathrm{h}$, while the measured travel time is 24 minutes, therefore 1 minute error is obtained. The results also show that if the driver waits for another 30 minutes he will be arriving to his destination point at 08:33 am instead of 8:10 am (leaving at 07:45 am), therefore he would have the choice whether or not it is worth arriving 23 minutes after or spending 7 minutes in traffic.

For the same scenario, figure 8 exhibits the predicted vehicle trajectory and velocity conditions for the highway section. The closeness between the predicted and measured

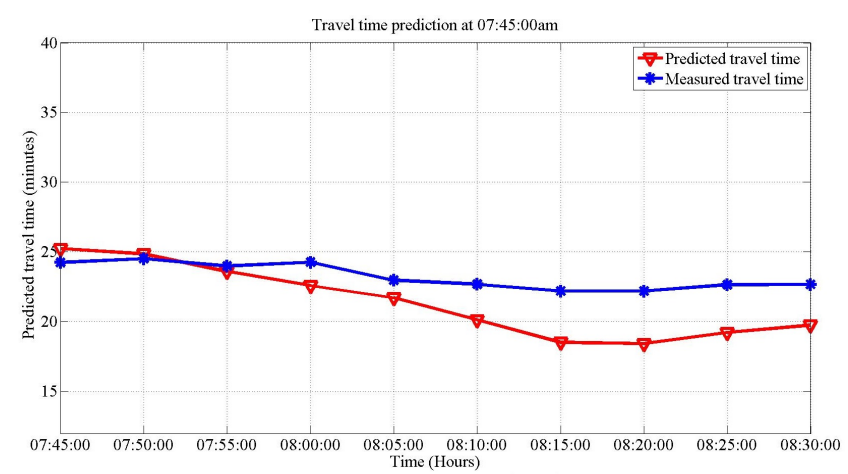

Fig. 7. Prediction result at 7:45 am.

trajectories can be clearly noticed. As it can be seen, these trajectories can change according to the driver departing time. For instance, between the kilometers 6 and 7 the congestion is dissipating as the departing time increases, it is observed that in this section a driver would encounter a mean velocity of $15 \mathrm{~km} / \mathrm{h}$ approximately when leaving at the current time, whereas 45 minutes later it would dissipate up to roughly $70 \mathrm{~km} / \mathrm{h}$. It is also seen that in the first 3.5 $\mathrm{km}$ the highway conditions are in free flow, with a velocity of $90 \mathrm{~km} / \mathrm{h}$. For the entire predicted velocity dynamics, the biggest congestion is obtained, as expected, in the end of the highway.

Intuitively figure 7 can be seen as the total time the driver would spend in the section chosen, while figure 8 would be the driving conditions experienced in the section.

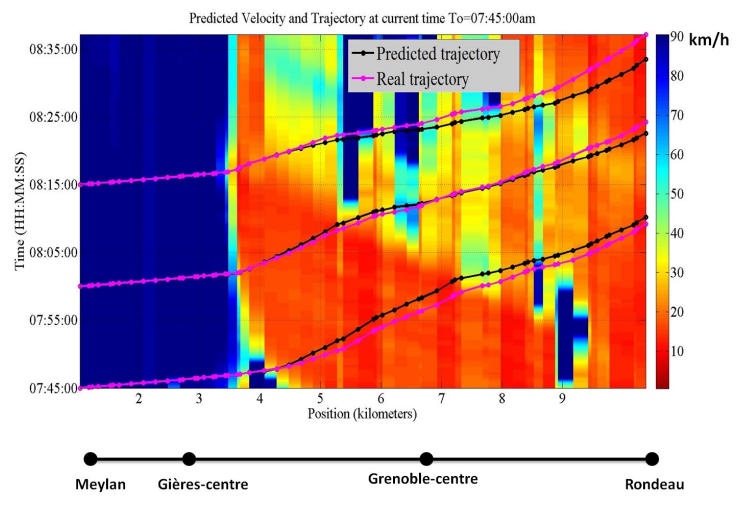

Fig. 8. Predicted vehicle trajectory moving through predicted velocity conditions at 7:45 am.

Considering now the current time at 19:00, figure 9 shows a good precision between the measured and predicted travel time. In this case we can note that driving conditions stay quite similar even when the horizon increases, which means that a driver would not gain much in waiting some minutes to depart. In contrast with the scenario before, in figure 10 a congestion is observed in both the beginning and in the end of the highway.

It is also seen from figure 10 that if a driver leaves at 19:00 between kilometer 4 and 5, he will encounter a 
strong congestion, whereas if he leaves 30 minutes later, for instance, this congestion will disappear.

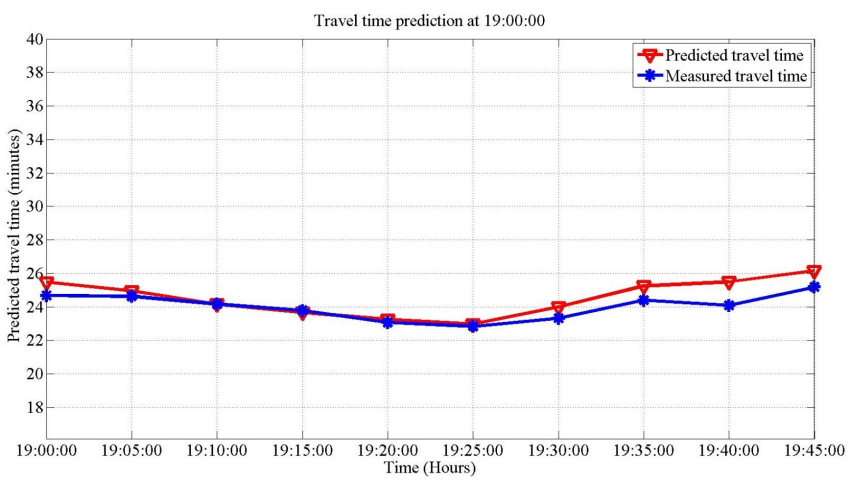

Fig. 9. Prediction result at 19:00.

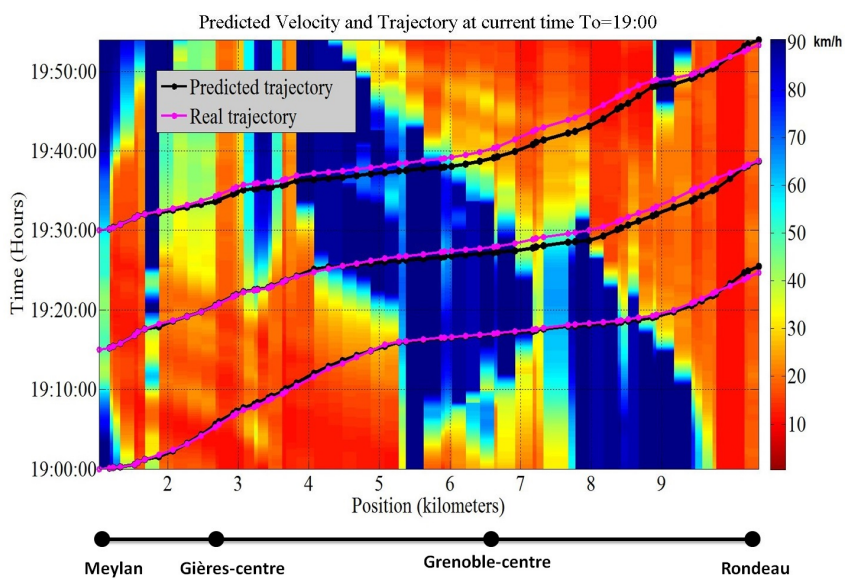

Fig. 10. Predicted vehicle trajectory moving through predicted velocity conditions at 19:00.

\section{CONCLUSIONS}

In this paper, we have addressed the problem of dynamic travel time forecasting by making use of flow and velocity measurements provided by sensors located at the node levels of the highway. We have focused our attention on a setup where new data arrive every few minutes or seconds, therefore the main benefit of this work is that under an online and no computational heavy methodology we can assess the prediction of travel time and traffic conditions. This forecasting approach was applied on Grenoble south ring. For this study case the simulation results show that the measured and predicted travel time agree for free-flow and congested period times, specially for smaller prediction horizons. As future research we are currently working on bounded travel time forecasting in the presence of uncertainties in the demand flows.

\section{ACKNOWLEDGEMENT}

The research leading to these results has received funding from the European Union Seventh Framework Programme
[FP7/2007-2013] under grant agreement no 257462 HYCON2 Network of excellence, and from the National Project Mesure et mOdelisation de la COgestion et de la POllution (MOCoPo).

\section{REFERENCES}

[1] D. Pisarski and C. Canudas-de Wit, "Analysis and design of equilibrium points for the cell-transmission traffic model," in Proc. of IEEE American Control Conference, Montreal, Canada, June 2012, pp. 5763-5768.

[2] — "Optimal balancing of road traffic density distributions for the cell transmission model," in Proc. of IEEE Conference on Decision and Control, Maui, Hawai, USA, December 2012, pp. 6969-6974.

[3] E. Vlahogiannia, J. Golias, and M. Karlaftisa, "Short term traffic forecasting: overview of objectives and methods," Transport Reviews, vol. 24, no. 5, pp. 553-557, September 2004.

[4] G. Hoffman and J. Janko, "Travel time as a basic part of the LISB guidance strategy," in Proc. of the Third International Conference on Road Traffic Control, London, England, June 1990, pp. 6-10.

[5] D. Park and L. Rilett, "Forecasting multiple-period freeway link travel times using modular neural networks," Transportation Research Record, vol. 1617, pp. 163-170, 1998.

[6] M. Chen and S. Chien, "Dynamic freeway travel time prediction using probe vehicle data: link-based vs. path-based," Transportation Research Board 80th Annual Meeting, 2001.

[7] S. Chien and C. Kuchipudi, "Dynamic travel time prediction with realtime and historical data," Transportation Research Board 81th Annual Meeting, 2002.

[8] J. Rice, "A simple and effective method for predicting travel times on freeways," IEEE Transactions on Intelling Transportation System, vol. 5, no. 3, pp. 200-207, September 2004.

[9] C. Wu, J. Ho, and D. Lee, "Travel-time prediction with support vector regression," IEEE Transactions on Intelligent Transportation Systems, vol. 5, pp. 276-281, 2004.

[10] J. Xia, Dynamic freeway travel time prediction using single loop detector and incident data. Kentucky (USA): University of Kentucky Doctoral Dissertations, 2006.

[11] X. Zhang and J. Rice, "Short-term travel time prediction," Transportation Research C, vol. 11, p. 210, 2003.

[12] D. Dailey, "Travel time estimates using a series of single loop volumen and occupancy measurements," Transportation Research Board 76th Annual Meeting, 1997.

[13] C. Canudas-de Wit, L. Leon, and A. Kibangou, "Graph constrainedCTM observer design for the Grenoble south ring," in Proc. of IFAC Symposium on Control in Transportation Systems, Sofia, Bulgaria, September 2012.

[14] R. Chrobok, J. Kaumann, and M. Schreckenberg, "Different methods of traffic forecast based on real data," European Journal of Operational Research, vol. 155, pp. 558-568, 2004.

[15] K. Myers and B. Tapley, "Adaptive sequential estimation with unknown noise statistics," IEEE Transactions on Automatic Control, vol. 21, no. 24, pp. 520-523, August 1976.

[16] L. Leon, A. Kibangou, and C. Canudas-de Wit, "Adaptive Kalman filtering for multi-step ahead traffic flow prediction," in Proc. of IEEE American Control Conference, Washington, DC, USA, June 2013.

[17] I.-C. Morarescu and C. Canudas-de Wit, "Highway traffic model-based density estimation," in Proc. of IEEE American Control Conference, San Francisco, CA, USA, June 2011.

[18] R. LeVeque, Numerical methods for conservation laws. Birkhäuser, 1992. 